\title{
Osmolaridade, ânion gap, potencial hidrogeniônico e íons plasmáticos mensuráveis de equinos Puro Sangue Árabe finalistas em provas de enduro de $90 \mathrm{~km}^{1}$
}

\author{
Cinthia B.S. Dumont ${ }^{2}$, Ceci R. Leite ${ }^{3}$, Júlia M. Moraes ${ }^{4}$, Meryonne Moreira ${ }^{5}$, \\ Augusto R.C. Moscardini ${ }^{6}$, Roberta F. Godoy ${ }^{2}$ e Eduardo M.M. Lima ${ }^{2 *}$
}

\begin{abstract}
Dumont C.B.S., Leite C.R., Moraes J.M., Moreira M., Moscardini A.R.C., Godoy R.F. \& Lima E.M.M. 2012. [Osmolality, anion gap, percentage Hydrogen (pH) and the mesasurable plasma ions in purebred Arabian horses which were finalists in $90 \mathrm{~km}$ endurance races.] Osmolaridade, ânion gap, potencial hidrogeniônico e íons plasmáticos mensuráveis de equinos Puro Sangue Árabe finalistas em provas de enduro de $90 \mathrm{~km}$. Pesquisa Veterinária Brasileira 32(6):542-546. Faculdade de Agronomia e Medicina Veterinária, Universidade de Brasília, ICC Ala Sul, Campus Darcy Ribeiro, Cx. Postal 4508, Brasília, DF 70760-701, Brazil. E-mail: limaemm@unb.br

We evaluated the behavior of osmolality, anion gap, $\mathrm{pH}$ and measurable plasma ions of 14 Purebred Arabian horses, 9 males and 5 females, finalists in an endurance race of $90 \mathrm{~km}$. In order to achieve this, we collected venous blood samples twice, at rest and after prolonged exercise, in order to measure the interest variables. The results allowed to observe that from the rest an increase with significant difference in the values of Hct, PPT and $\mathrm{AG}$, suggesting loss of fluids and slight dehydration. The $\mathrm{pH}$ and the $\mathrm{H}^{+}$ion almost had no changes, indicating absence of metabolic disorders. Reductions, with significant difference, were verified for the ions $\mathrm{Cl}^{-}, \mathrm{HCO}^{3}-, \mathrm{K}^{+}, \mathrm{Ca}^{++}$, as well as $\mathrm{BE}$. These data suggest that the type of exercise to which the animals were submitted was consistent with the athletic ability and that supplementation during the competition contributed to minimize such losses.
\end{abstract}

INDEX TERMS: Equine, exercise physiology, endurance, hydroelectrolytic balance.

RESUMO.- Foi avaliado o comportamento da osmolaridade, do ânion gap, do $\mathrm{pH}$ e dos íons plasmáticos mensuráveis de 14 equinos, 9 machos e 5 fêmeas, Puro Sangue Árabe finalistas em provas de enduro de $90 \mathrm{~km}$. Para tanto, foram colhidas em dois momentos (repouso e após o exercício prolongado) amostras de sangue venoso para a mensura-

\footnotetext{
${ }^{1}$ Recebido em 6 de julho de 2011.

Aceito para publicação em 15 de março de 2012

${ }^{2}$ Faculdade de Agronomia e Medicina Veterinária, Universidade de Brasília, Instituto Central de Ciências, Ala Sul, Campus Universitário Darcy Ribeiro, Cx. Postal 4508, Brasília, DF 70910-970, Brasil. *Autor para correspondência: limaemm@unb.br

${ }^{3}$ Faculdade de Veterinária, Universidade Federal Fluminense (UFF), Rua Vital Brazil 64, Niterói, RJ 24230340, Brasil.

${ }^{4}$ Departamento de Patologia Animal, Universidade Federal de Goiás (UFG), Campus Samambaia. Cx. Postal 131, Goiânia, GO 74001970, Brasil.

${ }^{5}$ 1o Regimento de Cavalaria de Guarda, Dragões da Independência, Brasília, DF.

${ }^{6}$ Regimento de Polícia Montada, Polícia Militar do Distrito Federal, Brasília, DF.
}

ção dessas variáveis. Pode-se verificar, a partir do repouso, aumento significativo dos valores do Hct, das PPT e do AG, sugerindo perda de fluidos e discreto grau de desidratação. $\mathrm{O} \mathrm{pH}$ e o íon $\mathrm{H}^{+}$quase não se alteraram, indicando ausência de distúrbios metabólicos. Reduções com diferenças significativas foram observadas para os íons $\mathrm{Cl}^{-}, \mathrm{HCO}^{3-}, \mathrm{K}^{+}$, $\mathrm{Ca}^{++}$, assim como do EB. Pode-se então, sugerir que o tipo de exercício a que os animais foram submetidos foi compatível com a capacidade atlética e ainda que a suplementação durante a competição contribuísse para minimizar tais perdas.

TERMOS DE INDEXAÇÃO: Equino, fisiologia do exercício, enduro, equilíbrio hidroeletrolítico.

\section{INTRODUÇÃo}

A ampla utilização da espécie equina ao longo dos tempos demonstra sua alta capacidade de reação às influências externas por meio de mecanismos adaptativos do organismo (Coenen 2005). Nesse contexto, o exercício é um fator de estresse que resulta em sobrecarga do sistema cardiovas- 
cular, aumento da mobilização de energia e termorregulação (Kronfeld 2001).

A água é o componente mais abundante no organismo e atua na diluição de inúmeras substâncias, entre elas os eletrólitos, que são de fundamental importância para a termorregulação a partir da transpiração (Coenen 2005). Assim, a sudação é a principal responsável pelos desequilíbrios hidroeletrolítico e ácido-base em equinos durante exercícios de longa duração, pois, nesse tipo de prova, os animais perdem entre $4 \%$ e $6 \%$ do peso corpóreo (Silva et al. 2009).

Os eletrólitos, no organismo animal, tem múltiplas funções, atuando na manutenção das forças osmóticas e favorecendo o equilíbrio de líquidos entre os compartimentos intra e extracelulares e na célula, desempenham funções básicas, tais como condução nervosa e despolarização de fibras musculares, tornando possível a contração muscular (Kronfeld 2001). Essas funções podem ser prejudicadas em resposta ao déficit de eletrólitos induzido pelo exercício, reduzindo assim a capacidade atlética (Coenen 2005). Pois, de fato, grandes reduções hidroeletrolíticas durante o exercício implicam desenvolvimento da síndrome de exaustão e outras desordens metabólicas (Schott II et al. 2006).

Os eletrólitos séricos são influenciados diretamente pela intensidade e duração do exercício, além da quantidade de perdas de fluidos pelo suor (Fernandes \& Larsson 2000, Ribeiro et al. 2004). Tais mudanças de concentração intra e extracelular de íons são de caráter transitório e ocorrem devido ao intercâmbio de íons entre o sangue e os tecidos (Martinez \& Ramón-Scaglione, 2000). Por isso, apesar de numerosos fatores contribuírem para o desenvolvimento de fadiga, o principal desafio para o desempenho constante durante exercício prolongado de resistência, é o progressivo esgotamento dos fluidos corporais e eletrólitos pelo organismo, que durante exercícios exaustivos pode levar a um aumento do risco de lesões e a vários problemas clínicos (Schott II et al. 2006).

Embora as alterações do status ácido-base, dos fluidos e do balanço eletrolítico em cavalos de enduro estejam bem definidas, a realização de estudos a campo, caracterizando a prova como laboratório em tempo real, se traduz como confirmação para os estudos controlados. Por isso, o presente trabalho teve como objetivo retratar e identificar as possíveis modificações dos íons mensuráveis, tais como sódio, potássio, cloro, cálcio ionizado, hidrogênio e bicarbonato, bem como, demonstrar o estabelecimento da osmolaridade e do ânion gap e do pH, em amostras de sangue venoso de equinos Puro Sangue Árabe finalistas em provas de enduro de $90 \mathrm{~km}$.

\section{MATERIAL E MÉTODOS}

Foram utilizados 14 equinos, 9 machos e 5 fêmeas, da raça Puro Sangue Árabe, com 6 a 12 anos de idade, submetidos a treinamento de enduro e participantes de campeonato regional de enduro da Federação Hípica de Brasília. Todos os animais tiveram a sua higidez comprovada por meio de avaliação clínica e ainda por meio de análise hematológica e de hemograma completo realizado no Medicalvet Laboratório Veterinário S.A, utilizando-se contador automático de células (Abacus Júnior Vet ${ }^{\circledR}$, Diagon, Ltda, Belo
Horizonte, Minas Gerais, Brasil). Os animais receberam diferentes tipos de alimentos e de suplementos eletrolíticos antes e durante o percurso. Os protocolos adotados obedeceram às normas do Comitê de Ética no Uso Animal da Universidade de Brasília sob o Protocolo no 88-2009.

As coletas de sangue venoso foram realizadas anaerobicamente, conforme descrito por Carlson (1997), utilizando-se seringas para coleta de sangue arterial e/ou venoso para gasometria $3 \mathrm{ml}$ 0,7x25mm (22G) (BD Preset Eclipse ${ }^{\circledR}$ ), coletando-se 1,6ml de sangue através da punção da veia jugular esquerda. As amostras foram identificadas e depositadas em banho de gelo por um período máximo de quatro horas até seu processamento. Sendo uma realizada em repouso (M0) e outra imediatamente após exercício prolongado de enduro (MF).

A avaliação no M0 foi obtida no próprio haras, em dias previamente estabelecidos, evitando que os animais fossem exercitados, sendo então, realizado exame clínico completo, coleta de sangue para quantificação dos parâmetros de interesse e determinação do peso corpóreo por meio da balança MGR 3000 Toledo. A avaliação no MF ocorreu no local de realização da prova, ao término do último anel da competição, cinco minutos após a inspeção veterinária oficial (vetcheck), seguindo a metodologia já empregada.

A análise do sangue coletado foi realizada em analisador automático de gases do Hospital Veterinário de Grandes Animais da Faculdade de Agronomia e Medicina Veterinária da Universidade de Brasília (Analisador de Gases Sanguíneos, Eletrólitos, Hemoglobina, Hematócrito e Saturação de $\mathrm{O}_{2}$ - OMNI C®-Roche Diagnóstica, Brasil) determinando-se: o logaritmo negativo da atividade de íons hidrogênio $(\mathrm{pH})$, as concentrações do íon cloro $\left(\mathrm{Cl}^{-}\right)$, íons $\mathrm{H}^{+}$e do bicarbonato $\left(\mathrm{HCO}^{3-}\right)$, valores do ânion gap (AG), do excesso/déficit de base (EB), da osmolaridade (Osm), as concentrações do íon sódio $\left(\mathrm{Na}^{+}\right)$, íon potássio $\left(\mathrm{K}^{+}\right)$e do cálcio ionizado $\left(\mathrm{Ca}^{++}\right)$.

Os resultados foram submetidos ao teste de normalidade Kolmogorov-Sminorv. Em seguida, os dados foram submetidos à aplicação do teste "T" de Student para se identificar a ocorrência de diferença estatística entre os parâmetros obtidos quando em repouso (M0) e imediatamente após exercício prolongado de enduro (MF), com nível de significância de 5\%. (Quadro 1)

\section{RESULTADOS E DISCUSSÃO}

Diante da análise da higidez dos animais, por meio das avaliações clínica e hematológica, foram observados os seguintes valores, em repouso e após o exercício respectivamente: frequência cardíaca (FC), 34,71 $\pm 3,50 \mathrm{bpm}$ e $51,78 \pm 6,08 \mathrm{bpm}$; temperatura retal $\left(\mathrm{TR}^{\circ}\right), 36,9 \pm 0,4^{\circ} \mathrm{C}$ e $38,0 \pm 0,6^{\circ} \mathrm{C}$; peso corporal, $377,00 \pm 23,64 \mathrm{kge} 369,85 \pm 26,63 \mathrm{~kg}$; hematócrito, $37,29 \pm 3,29 \%$ e 45,9 $\pm 7,08 \%$; proteína plasmática total, $7,10 \pm 0,24 \mathrm{~g} / \mathrm{dL}^{-1}$ e $7,60 \pm 0,59 \mathrm{~g} / \mathrm{dL}^{-1}$; plaquetas, $156714,29 \pm 38698,89 / \mathrm{mm}^{3}$ e $202000 \pm 45802,30 / \mathrm{mm}^{3}$; leucócitos, $8712,86 \pm 1616,54 \mathrm{~mm}^{-3} \mathrm{e}$ $14971,43 \pm 2297,30 \mathrm{~mm}^{-3}$.

Neste estudo, a perda de peso corpóreo foi de aproximadamente $2,2 \%$, com diferença estatística entre M0 e MF, coincidindo em parte com os achados de Filho et al. (2007), quando avaliou cavalos de alto desempenho praticantes de enduro, embora as perdas tenham sido discretamente menores daquelas observadas por Schott II et al. (2006), em cavalos que realizaram provas de $160 \mathrm{~km}$ de distância. Portanto isto sugeriu que a ocorrência de um processo de desidratação, pois para Schott II et al. (2006), a mensuração da perda de peso corporal foi uma forma confiável de se estimar a perda de fluidos por meio do suor durante exercícios 
Quadro 1. Valores das médias e desvios-padrão dos parâmetros obtidos por meio de exame hematológico e avaliação hidroeletrolítica de equinos PSA em repouso e no pós-exercício físico prolongado de enduro

\begin{tabular}{lcc}
\hline \multirow{2}{*}{ Parâmetros } & Repouso & Pós-exercício \\
\cline { 2 - 3 } & Média \pm DPM & Média \pm DPM \\
\hline PESO (Kg) & $382,50 \pm 24,42^{*}$ & $373,55 \pm 25,85^{*}$ \\
$\mathrm{Hct}(\%)$ & $37,9 \pm 2,5^{*}$ & $45,9 \pm 7,0^{*}$ \\
$\mathrm{PPT}(\mathrm{g} / \mathrm{dL})$ & $7,06 \pm 0,25^{*}$ & $7,60 \pm 0,54^{*}$ \\
$\mathrm{PH}$ & $7407,9 \pm 23,1$ & $7411,2 \pm 31,2$ \\
$\mathrm{Cl}(\mathrm{mmol} / \mathrm{L})$ & $100,11 \pm 2,84^{*}$ & $97,46 \pm 3,81^{*}$ \\
$\mathrm{H}^{+}(\mathrm{mmol} / \mathrm{L})$ & $39,14 \pm 2,11$ & $38,88 \pm 2,89$ \\
$\mathrm{HCO}(\mathrm{mmol} / \mathrm{L})$ & $29,27 \pm 1,56^{*}$ & $26,87 \pm 2,47^{*}$ \\
$\mathrm{AG}(\mathrm{mmol} / \mathrm{L})$ & $11,9 \pm 3,4^{*}$ & $16,39 \pm 2,078^{*}$ \\
$\mathrm{~EB}(\mathrm{mEq} / \mathrm{L})$ & $3,8 \pm 1,6^{*}$ & $1,91 \pm 2,59^{*}$ \\
$\mathrm{Osm}(\mathrm{mOsm} / \mathrm{L})$ & $273,36 \pm 4,23$ & $273,86 \pm 5,26$ \\
$\mathrm{Na}^{+}(\mathrm{mmol} / \mathrm{L})$ & $137,02 \pm 2,27$ & $137,30 \pm 2,8$ \\
$\mathrm{~K}^{+}(\mathrm{mmol} / \mathrm{L})$ & $4,34 \pm 0,33^{*}$ & $3,43 \pm 0,30^{*}$ \\
$\mathrm{Ca}^{++}(\mathrm{mmol} / \mathrm{L})$ & $1675,93 \pm 114,94^{*}$ & $1532,92 \pm 121,73^{*}$
\end{tabular}

Hct $=$ hematócrito, $\mathrm{PPT}=$ proteína plasmática total, $\mathrm{Cl}=$ íon cloro, $\mathrm{HCO}^{3}=$ íon bicarbonato, $\mathrm{AG}=$ ânion gap, $\mathrm{EB}=$ excesso de bases, $\mathrm{Osm}=$ osmolaridade, $\mathrm{Na}^{+}=$íon sódio, $\mathrm{K}^{+}=$íon potássio, $\mathrm{Ca}^{++}=$cálcio ionizado. A presença do asterisco $\left({ }^{*}\right)$ na mesma linha expressa diferença estatisticamente significante entre os momentos $(\mathrm{p}<0,05)$.

prolongados. No entanto, esse percentual reduzido indicou que o exercício realizado não promoveu maiores perdas ou ainda que estas tenham sido atenuadas pela suplementação oral com pasta eletrolítica oferecida pelos competidores (Teixeira-Neto et al. 2004).

O hematócrito (Hct) apresentou aumento significativo entre o MF e M0, corroborando com Robert et al. (2010) em equinos durante competições de enduro. Essa modificação poderia ser explicada pela contração esplênica, como foi observado por Ferraz et al. (2010) em equinos durante competições de pólo, ou pela desidratação, entretanto, as perdas substanciais de fluidos durante este tipo de atividade teriam maior influência na elevação do Hct em função da redução do volume plasmático (Kingston 2004). Os animais avaliados mantiveram-se com valores de Hct próximos aos fisiológicos (43 $\pm 4 \%$ ) conforme Rubio et al. (1995) para animais PSA acima de 4 anos, sugerindo portanto a ocorrência de um processo de desidratação leve sem interferências no desempenho.

Para Bayly \& Kline (2006), o aumento na concentração das proteínas plasmáticas (PPT) indicaria precisamente desidratação, sendo mais seguro que o Hct devido a contração esplênica. Nesse contexto, foi estabelecido que modificações nas concentrações de PPT, especialmente de albuminas, globulinas e fibrinogênio, ocorreriam em consequência do exercício, pois em equinos de enduro, este aumento poderia ser de até $25 \%$ (Bayly \& Kline 2006). Apesar de permanecerem com valores séricos normais, a contagem das PPT dos equinos aumentou significante $(\mathrm{p}=0,002)$, entre os momentos avaliados, coincidindo com Filho et al. (2007) e Robert et al. (2010). Ainda neste contexto, Martinez \& Ramón-Scaglione (2000) relataram aumento progressivo das proteínas plasmáticas ao longo da prova, aumento do Hct e manifestações relativas de fadiga e desidratação, diferindo do observado nos finalistas, pois a elevação das PPT foi discreta, mantendo-se próxima ao fi- siológico (5,5-7,5g/dL) (Rose \& Hodgson 1994), sobretudo inferior aos 25\% aceitos por Bayly \& Kline (2006).

Nos animais avaliados, o pH manteve-se dentro dos valores de referência, entre 7,32 e 7,44, conforme descrito por Carlson (1997), sugerindo que as perdas de $\mathrm{Cl}^{-}$não foram suficientes para induzir alcalose metabólica hipoclorêmica, que, seria uma complicação importante na síndrome de exaustão. Mesmo observando que os valores do íon cloro $\left(\mathrm{Cl}^{-}\right)$diminuíram significativamente $(\mathrm{p}=0,048)$ entre as duas avaliações, corroborando Robert et al. (2010) e Viu et al. (2010), diferindo de Ferraz et al. (2010), tendo em vista que a intensidade do esforço teria sido distinta, em função do exercício de pólo se tratar de uma atividade de alta intensidade e curta duração . No entanto, esses valores permaneceram dentro do considerado fisiológico (94-104mmol/L) (Rose \& Hodgson 1994) e (95-111mmol/L) (Thomassian 2005). Em relação ao íon $\mathrm{H}^{+}$, não foi observada diferença estatística entre o M0 e o MF, e esteve ainda dentro dos valores de referência, 32 e $44 \mathrm{mmol} / \mathrm{L}$ no meio extracelular (Carlson 1997, Thomassian 2005). Tal achado diferiu dos estudos de Ferraz et al. (2010), devido ao aumento da produção de prótons $\mathrm{H}^{+}$em função da atividade realizada. Sugerindo assim, que os animais estavam adaptados ao exercício realizado pois, não foi capaz de modificar o equilíbrio ácido base e nem hipovolemia decorrente de desidratação. Este dado sugere ainda que até o momento da avaliação, houve uma resposta respiratória compensatória, que foi capaz de corrigir distúrbios metabólicos leves (Fettman 2004). Em relação ao bicarbonato $\left(\mathrm{HCO}^{3-}\right)$ pode-se ver uma redução da sua concentração, entre os momentos MF e M0, com diferença estatística, corroborando Ferraz, et al. (2010), embora este pesquisador tenha avaliado equinos em competições de pólo equestre, sugerindo que os animais avaliados neste estudo estivessem desenvolvendo acidose metabólica discreta, porém, os valores mantiveram-se dentro do considerado fisiológico, ou seja, $29,5 \pm 2,4 \mathrm{mmol} / \mathrm{L}$ (Carlson 1997), 24 a 30mmol/L (Kingston 2004) e 20 e 28mmol/L (Thomassian 2005), respectivamente. Portanto, sem manifestação de distúrbios metabólicos. Revelando assim, condicionamento adequado ao tipo de exercício a que os animais foram submetidos. Ainda neste contexto, podemos sugerir que o aumento da intensidade do mesmo, resultaria em acidose metabólica.

Foi observada diferença estatística com aumento $(p<0,001)$ absoluto, entre as duas avaliações em relação ao $\mathrm{AG}$, representado pela equação: $\mathrm{AG}=\left(\mathrm{Na}^{+}+\mathrm{K}^{+}\right)-\left(\mathrm{Cl}^{-}+\mathrm{HCO}^{3-}\right.$ ). A elevação dessa variável, que tem como valores fisiológicos entre 6 e 15mmol/L (Thomassian 2005), esteve associada à acidose láctica decorrente de exercícios anaeróbios com acúmulo de lactato, à desidratação, à acidose orgânica, à insuficiência renal (retenção de ácidos) e à hipovolemia (Carlson 1997). 0 discreto aumento observado neste estudo ao final do exercício prolongado de enduro apresentou relação inversa com o $\mathrm{HCO}^{3-}$, perfil que foi semelhante ao relatado por Costa et al. (2008), e poderia sugerir um grau leve de desidratação com ausência de significância clínica, tendo em vista que os animais analisados foram finalistas.

Dentre os animais avaliados neste estudo, foi observado que a mensuração do EB apresentou diferença estatística 
$(\mathrm{p}=0,028)$, entre os dois momentos, com valores dentro da variação fisiológica citada por Mello (2007), -4 a $+4 \mathrm{mEq} / \mathrm{L}$, com os valores no $\mathrm{M} 0$ similares àqueles obtidos por esse pesquisador no tempo 0 , também em equinos hígidos, embora não atletas. Entretanto, os valores diferiram daqueles obtidos por Di Filippo et al. (2009) em animais PSA, tanto em repouso como após o exercício, e também diferentes dos encontrados por Lekeux \& Art (1995) em animais de corrida, em repouso e após exercício. Em função das variações individuais dos parâmetros que influenciariam na determinação do EB, pode-se sugerir que as divergências entre os autores tenham ocorrido em função das características do tipo de exercício, nível de condicionamento e tipo de nutrição. Pois a variação normal seria de $-2 \mathrm{a}+2 \mathrm{mEq} / \mathrm{L}$ para Carlson (1997) e Thomassian (2005) e de -6 a $+6 \mathrm{mEq} / \mathrm{L}$ para Kingston (2004) e Viu et al. (2010).

Nesse estudo, a mensuração da osmolaridade plasmática não apresentou diferença estatística entre o M0 e o MF, acompanhando o comportamento do sódio, visto que esse cátion seria o principal regulador dessa variável no organismo corroborando assim achados de Silva et al. (2009). Pois conforme Verbalis (2003), embora o excesso de sódio pudesse causar um estado de hiperosmolaridade secundária à hipernatremia, na grande maioria dos casos, isso foi decorrente da excessiva perda de água associada à diminuição da ingestão de água ou excreção excessiva (sudação, diarréia e refluxo). Sugerindo que as perdas de fluidos através da sudação não foram significativas para influenciar esse parâmetro.

Com relação ao íon sódio, não houve diferença estatística entre os momentos, sendo que estes ainda mantiveram-se dentro dos valores fisiológicos (135-145mmol/L) (Rose \& Post 2001) e (132-155mmol/L) (Thomassian 2005). De forma semelhante às afirmações de Martínez \& Ramón-Scaglione (2000), Teixeira-Neto et al. (2004) e Di Filippo et al. (2009), quando as concentrações sanguíneas deste íon geralmente não se alteraram durante o exercício, seja máximo, seja submáximo, afirmativa que, por sua vez, foi confirmada por achados de Filho et al. (2007). Determinando assim o ideal equilíbrio iônico que os animais finalistas apresentavam ao final da prova, bem como, seu adequado preparo em relação ao esforço. $\mathrm{O}$ íon $\mathrm{K}^{+}$apresentou diferença estatística $(\mathrm{p}<0,001)$, com redução da concentração plasmática após o exercício, bem como, o evidenciado por Filho et al. (2007), pois esse íon foi liberado no plasma pelos miócitos durante exercícios intensos conforme observado por Ferraz et al. (2010) e, na fase inicial, nos exercícios de resistência, aumentou sua concentração plasmática, que, ao final, diminuiu quando comparada aos valores basais em função das perdas desse eletrólito através da sudação, seu retorno aos miócitos e excreção renal (Hess et al. 2005). Nos animais avaliados, os valores permaneceram com concentração sérica dentro dos valores considerados fisiológicos para o equino (3,0-5,0mmol/l) (Seahorn \& Seahorn 2003).

Em relação ao cálcio ionizável $\left(\mathrm{Ca}^{++}\right)$foi possível verificar que os valores séricos neste estudo foram semelhantes aos descritos na literatura consultada, ou seja, de 1,54 a 1,69 mmol/l (Berlin \& Aroch 2009), mas com diferença estatística $(p=0,004)$, com valores menores no MF seme- lhante aos achados de Di Filippo et al. (2009), que tiveram como provável origem a perda no suor. Outra explicação que justificaria essa redução seria o movimento do cálcio para o meio intracelular permitindo a contração muscular durante o exercício, pois este íon liga-se com a molécula de troponina visando à ativação actina - miosina, sendo assim a manutenção da contração muscular a sua principal função durante o exercício (McKeever 2004).

\section{CONCLUSÕES}

Foi possível concluir com este estudo que a metodologia empregada constituiu um meio de avaliação do condicionamento de boa acurácia e simples realização.

Os resultados sugeriram discreto grau de desidratação compatível com a perda de fluidos e de eletrólitos por meio da sudação característica nesse tipo de atividade.

Tais perdas resultaram em reduções significativas dos íons $\mathrm{Cl}^{-}, \mathrm{HCO}^{3}-, \mathrm{K}^{+}, \mathrm{Ca}^{++}$, assim como do EB. Entretanto, mantiveram-se dentro dos valores de referência citados na literatura consultada.

Os dados indicaram ainda ausência de distúrbios metabólicos, frente aos dados obtidos para o $\mathrm{pH}$ e o íon $\mathrm{H}^{+}$, quase não terem sofrido alterações.

É possível considerar que os animais avaliados não tenham sofrido perdas suficientes para o desenvolvimento de desequilíbrios metabólicos que induzissem manifestações clínicas que comprometessem o desempenho atlético, tendo em vista que foram finalistas.

Contudo, pode-se sugerir que os animais estavam adaptados ao tipo de exercício a que foram submetidos e ainda que a suplementação, através do fornecimento de forragem, de soluções eletrolíticas e de água durante o percurso, contribuiu para minimizar tais perdas.

\section{REFERÊNCIAS}

Bayly W.M. \& Kline K.A. 2006. Hematología y bioquímica, p.145-151. In: Boffi F.M. (Ed.), Fisiología del Ejercicio en Equinos. Inter-Médica, Buenos Aires.

Berlin D. \& Aroch I. 2009. Concentrations of ionized and total magnesium and calcium in healthy horses: Effects of age, pregnancy, lactation, $\mathrm{pH}$ and sample type. Vet. Journal 181(3):305-311.

Carlson G.P. 1997. Fluid, electrolyte, and acid-base balance, p.485-516. In: Kaneko J.J., Harvey J.W. \& Bruss M.L. (Eds), Clinical Biochemistry of Domestic Animals. $5^{\text {th }}$ ed. Academic Press, San Diego.

Coenen M. 2005. Exercise and stress: impact on adaptive processes involving water and electrolytes. Livest. Prod. Sci. 92:131-145.

Costa N.S., Ribeiro G., Dória R.G.S., Canola P.A., Silva P.C., Jorge R.L.N. \& Fagliari J.J. 2008. Hemograma e hemogasometria de equinos submetidos à obstrução experimental de jejuno. Arq. Bras. Med. Vet. Zootec. 60(6):1367-1374.

Di Filippo P.A., Gomide L.M.W., Orozco C.A.G., Giannocaro M.A., Martins C.B., Lacerda-Neto J.C. \& Santana A.E. 2009. Alterações hemogasométricas e hidroeletrolíticas de cavalos da raça árabe durante prova de enduro de $60 \mathrm{~km}$. Ciênc. Anim. Bras. 10:840-846.

Fernandes W.R. \& Larsson M.H.M.A. 2000. Alterações nas concentrações séricas de glicose, sódio, potássio, uréia e creatinina, em equinos submetidos a provas de enduro de $30 \mathrm{~km}$ com velocidade controlada. Ciência Rural 30:393-398.

Ferraz G.C., Soares O.A.B., Foz N.S.B., Prereira M.C. \& Queiroz-Neto A. 2010. The workload and plasma íon concentration in a training match session of high-goal (elite) polo ponies. Equine Vet. J. 42(38):191-195. 
Fettman M.J. 2004. Fluid and electrolyte metabolism, p.329-355. In: Thrall M.A., Baker D.C., Campbell T.W., Lassen E.D., De Nicola D.B., Rebar A., Fettman M.J. \& Weiser G. (Eds), Veterinary Hematology and Clinical Chemistry. Lippincott Williams and Wilkins, Philadelphia.

Filho J.N.P.P., Neto T.L.B., Rodrigues P.H.M. \& Garcia H.P.L. 2007. Parâmetros fisiológicos do desempenho de cavalos de alta performance hidratados voluntariamente com água ou solução isotônica contendo carboidrato. Brazilian J. Vet. Res. Animal Sci. 44(2):122-131.

Hess T.M., Kronfeld D.S., Williams C.A., Waldron J.N., Graham-Thiers P.M., Greiwe-Crandell K., Lopes M.A. \& Harris M.A. 2005. Effects of oral potassium supplementation on acide-base status and plasma ion concentrations of horses during endurance exercise. Am. J. Vet. Res. 66:466-473.

Kingston J.K. 2004. Hematologic and serum biochemical responses to exercise and training, p.939-948. In: Hinchcliff K.W., Kaneps A.J. \& Geor R.J. (Eds), Equine Sports Medicine and Surgery: Basic and clinical sciences of the equine athlete. W.B. Saunders, Philadelphia.

Kronfeld D.S. 2001. Body fluids and exercise: Physiological responses (Part I). J. Equine Vet. Sci. 21(7):312-322.

Lekeux P. \& Art T. 1994. The respiratory system: anatomy, physiology, and adaptations to exercise and training, p.81-127. In: Hodgson D.R. \& Rose R.J. (Eds), The Athletic Horse. W.B. Saunders, Philadelphia.

Martínez P. \& Ramón-Scaglione M.M.C. 2000. Cambios sanguíneos y sudorales em equinos sometidos a carreras de resistencia. Avances en Cienc. Vet. 15:19-30.

McKeever K.H. 2004. Physiology of body fluids in the equine athlete, p.853-871. In: Hinchcliff K.W., Kaneps A.J. \& Geor R.J. (Eds), Equine Sports Medicine and Surgery: Basic and clinical sciences of the equine athlete. W.B. Saunders, Philadelphia.

Ribeiro C.R., Martins E.A., Ribas J.A. \& Germinaro A. 2004. Avaliação de constituintes séricos em equinos e muares submetidos à prova de resistência de 76 km, no pantanal do Mato Grosso, Brasil. Ciência Rural 34:1081-1086.

Robert C., Goachet A.G., Fraipont A., Votion D.M., Van Erck E. \& Leclerc J.L.
2010. Hydration and electrolyte balance in horses during an endurance season. Equine Vet. J. (Suppl.) 38:98-104.

Rose B.D. \& Post T.W. 2001. Clinical Physiology of Acid-Base and Electrolyte Disorders. $5^{\text {th }}$ ed. McGraw-Hill, New York. 992p.

Rose R.J. \& Hodgson D.R. 1994. Clinical exercise testing, p.245-257. In: Hodgson D.R. \& Rose R.J. (Eds), The Athletic Horse: Principles and practice of equine sports medicine. W.B. Saunders, Philadelphia.

Rubio M.D., Muñoz A., Santisteban R. \& Catejón F.M. 1995. Comparative hematological study of two breeds of foals (Andalusian and Arab) subjected to exercise of progressive intensity. J. Vet. Med. Sci. 57:311-315.

Scoth II H.C., Marlin D.J., Geor R.J., Holbrooks T.C., Deaton C.M., Vicent T., Dacrey K., Schroter R.C., Jose-Cunilleras E. \& Cornelisse C.J. 2006. Changes in selected physiological and laboratory measurements in elite horses competing in a $160 \mathrm{~km}$ endurance ride. Equine Vet. J. 36(Suppl.):37-42.

Seahorn J.L. \& Seahorn T.L. 2003. Fluid theraphy in horses with gastrointestinal disease. Vet. Clin. North Am., Equine Pract. 19(3):665-679.

Silva M.A.G., Martins C.B., Gomide L.M.W., Albernaz R.M., Queiroz-Neto A. \& Lacerda-Neto J.C. 2009. Determinação de eletrólitos, gases sanguíneos, osmolalidade, hematócrito, hemoglobina, base titulável e anion gap no sangue venoso de equinos destreinados submetidos a exercício máximo e submáximo em esteira rolante silva. Arq. Bras. Med. Vet. Zootec. 61(5):1021-1027.

Teixeira-Neto A.R., Ferraz G.C., Mataqueiro M.I., Lacerda-Neto J.C. \& Queiroz-Neto A. 2004. Reposição eletrolítica sobre variáveis fisiológicas de cavalos em provas de enduro de 30 e $60 \mathrm{Km}$. Ciência Rural 34(5):1505-1511.

Thomassian A. 2005. Enfermidade dos Cavalos. $4^{\text {th }}$ ed. Livraria Varela, São Paulo. 65p.

Verbalis J.G. 2003. Disorders of body water homeostasis: Best practice 96. Res. Clin. Endocrinol. Metabol. 17(4):471-503.

Viu J., Jose-Cunilleras E., Armengou L., Cesarini C., Taracón I., Rios J. \& Monreal L. 2010. Acid-base imbalances during a $120 \mathrm{~km}$ endurance race compared by tradicional and simplified strong ion difference methods. Equine Vet. J. 38(Suppl.):76-82. 